\title{
Radiation pressure on non-spherical dust grains in envelopes of late-type giants
}

\author{
V.B. Il'in ${ }^{1,2}$ and N.V. Voshchinnikov ${ }^{2}$ \\ 1 Max Planck Society, Research Unit "Dust in Star-Forming Regions", Schillergäßchen 3, D-07745 Jena, Germany \\ 2 Astronomical Institute, St. Petersburg University, St. Petersburg 198904, Russia
}

Received April 28; accepted July 10, 1997

\begin{abstract}
Stellar winds are typical features of the AGB evolution. They are formed as the result of the radiation pressure on dust grains.

We calculate the radiation pressure force of a red giant acting on prolate and oblate spheroidal grains of different size, aspect ratio and chemical composition. The exact solution to the light scattering problem for spheroids is used. The force and the grain drift velocity (relative to gas) are compared for spheroids and spheres of the same volume.
\end{abstract}

It is found that for small spheroids (radii of equivolume sphere $r_{V} \lesssim 0.1 \mu \mathrm{m}$ ) the radiation pressure force usually is greater than that for spheres. A very significant effect occurs for strongly absorbing particles with $r_{V} \lesssim 0.03-0.05 \mu \mathrm{m}$. It is caused by the resonance absorption of incident radiation whose electric vector is parallel to the major axis of a particle. As a result, the velocity of a sphere and equivolume spheroid of iron can differ in $5-10$ times or more.

Another effect is the deviations of the radiation pressure force from the direction of the wave-vector of incident radiation. This is due to an azimuthal asymmetry of geometry of light scattering by non-spherical particles. The transversal component of the force is more important for dielectric particles and can reach up to $30-50 \%$ of the radial one for silicate grains of the size $r_{V} \approx 0.3 \mu \mathrm{m}$. It should increase the path of non-spherical grains in stellar envelopes and the number of dust-gas collisions in the comparison with spherical grains.

The tendency of a strengthening of the radial component of the radiation pressure force and a weakness of the transversal one with a decrease of the stellar effective temperature is noted.

Key words: circumstellar matter - stars: AGB, postAGB

Send offprint requests to: N.V. Voshchinnikov

\section{Introduction}

The extended envelopes around cool giant stars are believed to be a place of dust formation and important sources of recycled interstellar matter. Though the single mechanism initiating the wind from evolved stars is not acknowledged yet, it is generally accepted that the mass loss is controlled by radiation pressure on dust grains (Lafon \& Berruyer 1991; Winters 1994). Momentum of stellar radiation is transferred to the circumstellar gas through radiation pressure on the grains and gas-grain collisions. Recently, Netzer \& Elitzur (1993) and Habing et al. (1994) have connected the rate of mass loss from cool giants with the observed gas outflow velocity.

The shape of particles should be an important factor that affects the momentum transfer. However, this effect has been considered only in a few cases. Voshchinnikov \& Il'in (1983b,c) investigated the pressure of radiation of different stars on infinite circular cylinders. Il'in (1994) analyzed the motion of very small spheroidal grains in the envelopes of $\mathrm{M}$ giants.

The fact that starlight is polarized in many lines of sight indicates that interstellar grains are non-spherical and aligned. As neither theoretical nor observational arguments exist in favor of changes of particles shapes in the diffuse interstellar medium, we can propose that the shape of grains originating in the envelopes of evolved stars differ from the spherical one.

An indirect indication that the non-spherical grains are present in the envelopes comes from the observed time and wavelength variations of the positional angle of polarization of the red giants. The difference of its values in the blue and red bands reaches $20^{\circ}-60^{\circ}$ even after the subtraction of interstellar polarization (Dyck \& Jennings 1971; Shawl 1975). This behaviour is hard to explain within the model of a single star with the envelope populated by spherical grains only. On the other hand, variations of the degree and the direction of orientation of non-spherical circumstellar grains could naturally produce the effect. 
In this paper, we consider how the particle's shape can affect the grain motion in the vicinities of late-type stars. The radiation pressure force is calculated for prolate and oblate spheroids of different semiaxes ratio, size and composition. The force and the drift velocity obtained from the solution of a simple equation of dust motion are compared with those for spheres of the same volume. The model and equations used are presented in Sects. 2 and 3, the results of calculations are discussed in Sect. 4, and the conclusions are given in Sect. 5.

\section{Model}

\subsection{Spectral energy distribution of stellar radiation}

Radiation pressure on a dust grain is the result of absorption and scattering of photons of different energies. The observed spectra of late-type stars are filled with a lot of molecular features but for the sake of simplicity we approximate the spectral energy distribution by the Planck function with the effective temperature of the star. Note that such an approach may be not exact, in particular for the stars with chromospheres. Furthermore, in the outer parts of the optically thick envelopes, the peak of the energy distribution should shift to longer wavelengths (see, e.g., Netzer \& Elitzur 1993; Bagnulo et al. 1995). Nevertheless, in general our skipping the details of stellar spectra does not strongly affect the results of the calculations as radiation pressure on the particles of different shape is mainly compared.

The effective temperatures of late-type giants and supergiants lie in the range of $T_{\star} \approx 2000-3500 \mathrm{~K}$ (Pégourié 1987; Lorenz-Martins \& Lefèvre 1994). Below, the value of $T_{\star}=2500 \mathrm{~K}$ is used in calculations. Some effects of stellar temperature variations are discussed in Sect. 4.4.

\subsection{Dust grains}

\subsubsection{Chemical composition}

Amorphous carbon and amorphous silicates are commonly considered as the major sources of dust emission observed in spectra of carbon-rich and oxygen-rich stars, respectively. The evidence in favor of these materials came from both theoretical modelling (Gail \& Sedlmayr 1984) and laboratory analysis (Jäger et al. 1994).

It is a hard task to determine a concrete type of the carbonaceous or silicate material. Moreover, it is evident that a mixture of different dusty components should coexist in the envelopes. For example, silicon carbide, silicate sulfide and even silicate features have been detected in spectra of some carbon-rich stars (Baron et al. 1987; Goebel \& Moseley 1985; Little-Marenin 1986). Some emission bands found in oxygen-rich spectra have been tentatively identified with crystalline forms of silicates (Waters et al. 1996). At last, iron and oxidic particles can also condense in the circumstellar shells irrespective of the $\mathrm{C} / \mathrm{O}$ ratio.
Amorphous carbon and astronomical silicate have been chosen as the basic species in our modelling. The optical constants of these species were taken from the papers of Rouleau \& Martin (1991; their AC1 table) and Laor \& Draine (1993). We have been also considered other materials: iron (optical constants from Ordal et al. 1988 and Pollack et al. 1994) and magnetite (Huffman \& Stapp 1973; Steyer 1974) as examples of highly absorbing materials and artificial dirty silicate proposed by Ossenkopf et al. (1992; OHM-silicate) and clean glassy pyroxene with the ratio $\mathrm{Fe} / \mathrm{Mg}=0.2$ (Dorschner et al. 1995) as examples of different types of silicates. Note that the latter two species cover almost completely the range of possible dirtiness of circumstellar silicates (Jones \& Merrill 1976; David \& Pégourié 1995).

\subsubsection{Shape}

So far only the formation of spherical grains in the envelopes of late-type stars has been considered (e.g., Draine 1981; Gail \& Sedlmayr 1985; Fadeyev 1987; Fleischer et al. 1992; Cadwell et al. 1994 and references therein). The theory of nucleation and growth of non-spherical particles is still in the period of infancy.

We suppose that the circumstellar grains are prolate or oblate homogeneous spheroids with the aspect ratio $a / b$ ( $a$ and $b$ are the major and minor semiaxes of a spheroid, respectively). Note that variations of $a / b$ allow to cover a wide set of the particle's shapes from disks to needles.

\subsubsection{Size}

Dust grains condense and grow in the envelopes of latetype stars. The size spectrum of the grains extends from tiny particles to those with radii up to $1 \mu \mathrm{m}$ or more (see discussion in Lafon \& Berruyer 1991). The upper cut-off of the size distribution is the subject of the controversy. However, the models developed to fit available observational data show that on average the size of grains in oxygen-rich giants should be larger than that in carbonrich stars (Jura 1994, 1996; Bagnulo et al. 1995).

To compare the particles of the same volume but of different shapes, it is suitable to characterize their size by the radius $r_{V}$ of the sphere whose volume is equal to that of a spheroid. The major semiaxis of the spheroid is connected with $r_{V}$ as follows:

$$
a=r_{V}\left(\frac{a}{b}\right)^{2 / 3}
$$

for prolate spheroids and

$a=r_{V}\left(\frac{a}{b}\right)^{1 / 3}$

for oblate ones. In our calculations, the particles with the size $r_{V}=0.01-0.5 \mu \mathrm{m}$ are considered. 


\subsubsection{Structure}

In principle, the grains growing in the envelopes may be fluffy (or porous). We use a standard approach to model this effect (see, e.g., Mukai et al. 1992). The Bruggemann mixing rule (Bohren \& Huffman 1983) is applied to construct a mean, effective dielectric function $\varepsilon_{\text {eff }}$ of an aggregate composed of $n$ materials with dielectric functions $\varepsilon_{i}$

$\sum_{i=1}^{n} f_{i} \frac{\varepsilon_{i}-\varepsilon_{\mathrm{eff}}}{\varepsilon_{i}+2 \varepsilon_{\mathrm{eff}}}=0$

where $f_{i}$ is the volume fraction occupied by the constituent material of the type $i$. The radiation pressure force is calculated for compact particles with the mean function $\varepsilon_{\text {eff }}$. We consider spheroids consisting of vacuum $(\varepsilon=1)$ and silicate or carbon with the vacuum filling factor $f$ from 0 up to 0.9 .

\subsection{Grain alignment}

Collisions with the surrounding gas atoms and molecules cause rotation of dust grains with high angular velocities $\left(>10^{5} \mathrm{~s}^{-1}\right)$. Interstellar grains are believed to rotate around their axes of maximum moment of inertia, and on average the angular velocity vectors of the grains are parallel to the magnetic fields (Spitzer 1978). Circumstellar dust grains may be aligned under the action of the anisotropic fluxes of radiation or gas (Dolginov et al. 1979). Apparently, the radial motion of the grains should lead to their rotation in the planes containing the radius-vector. However, non-radial gaseous fluxes or spiral circumstellar magnetic fields arising as a result of stellar rotation (e.g., Woitke et al. 1993) may lead to other orientations of grains.

In our modelling, two types of grain alignment are considered: grains arbitrarily oriented in space (3Dorientaion) and in a plane (2D-orientaion or perfect rotational orientation). In the last case, the major axis of a rotating spheroid remains in the same plane. The angle between the angular velocity of the grain and the wave-vector of incident radiation $\Omega$ is an input parameter $\left(0^{\circ} \leq \Omega \leq 90^{\circ}\right)$.

\section{Basic equations}

\subsection{Radiation pressure force}

\subsubsection{Nonrotating grains}

An azimuthal asymmetry of the geometry of the light scattering by non-spherical particles (see Fig. 1) provokes the non-coincidence of the directions of the radiation pressure force and of the wave-vector of incident radiation. This fact was mentioned in the literature (e.g., Kerker 1981)

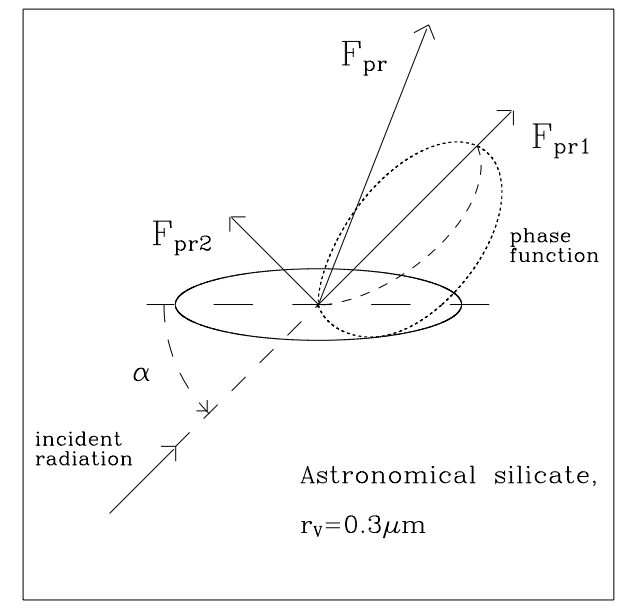

Fig. 1. Geometry of light scattering by a prolate spheroid with $a / b=4$. The wave-vector of incident non-polarized radiation $(\lambda=1 \mu \mathrm{m})$ forms the angle $\alpha=45^{\circ}$ with the rotation axis of spheroid. Short-dashed curve shows the angular distribution of the scattered radiation (phase function). The part of scattered radiation which is symmetric relative to the direction of incident radiation is plotted by long-dashed curve. The directions of the resulting radiation force as well as its components in the radial and transversal directions are indicated

but the detailed consideration has been made only for infinite cylinders (Voshchinnikov \& Il'in 1983a) and spheroids (Voshchinnikov 1990).

The radiation pressure force on a non-spherical particle may be written as follows:

$\mathbf{F}_{\mathrm{pr}}=\frac{1}{c} \int_{0}^{\infty}\left(C_{\mathrm{pr} 1} H_{\nu} \boldsymbol{r}_{0}+C_{\mathrm{pr} 2} H_{\nu} \boldsymbol{t}_{0}\right) \mathrm{d} \nu$

where $H_{\nu}$ is the flux of non-polarized radiation of a point source at frequency $\nu, c$ the velocity of light, $\boldsymbol{r}_{0}$ and $\boldsymbol{t}_{0}$ are the unit vectors in the radial and transversal directions $\left(\boldsymbol{r}_{0} \| \boldsymbol{k}, \boldsymbol{t}_{0} \perp \boldsymbol{k}, \boldsymbol{k}\right.$ is the wave-vector of incident radiation), and $C_{\mathrm{pr} 1,2}$ the radiation pressure cross-sections for the corresponding directions.

We approximate the flux $H_{\nu}$ in Eq. (4) by the Planck function with the effective temperature of the star $\pi B_{\lambda}\left(T_{\star}\right)$ and consider the Planck mean cross-sections and efficiency factors

$\bar{C}_{\mathrm{pr} 1,2}=\frac{1}{2}\left(\bar{Q}_{\mathrm{pr} 1,2}^{\mathrm{TM}}+\bar{Q}_{\mathrm{pr} 1,2}^{\mathrm{TE}}\right) G$

$\bar{Q}_{\mathrm{pr} 1,2}^{\mathrm{TM}, \mathrm{TE}}\left(r_{V}, a / b, \alpha, T_{\star}\right)=$
$\frac{1}{\sigma T_{\star}^{4}} \int_{0}^{\infty} Q_{\mathrm{pr} 1,2}^{\mathrm{TM}, \mathrm{TE}}\left(m_{\lambda}, x_{V}, a / b, \alpha\right) \pi B_{\lambda}\left(T_{\star}\right) \mathrm{d} \lambda$.

Here, $\sigma$ is the Stephan-Boltzmann constant, $m_{\lambda}$ the refractive index of the grain material, $x_{V}=2 \pi r_{V} / \lambda$ the size parameter, $\alpha$ the angle between the rotation axis of the spheroid and the wave-vector $\left(0^{\circ} \leq \alpha \leq 90^{\circ}\right), G$ the 
geometrical cross-section of a spheroid (the area of the particle shadow) that is

$G(\alpha)=\pi r_{V}^{2}\left(\frac{a}{b}\right)^{-2 / 3}\left[\left(\frac{a}{b}\right)^{2} \sin ^{2} \alpha+\cos ^{2} \alpha\right]^{1 / 2}$

for an prolate spheroid and

$G(\alpha)=\pi r_{V}^{2}\left(\frac{a}{b}\right)^{2 / 3}\left[\left(\frac{a}{b}\right)^{-2} \sin ^{2} \alpha+\cos ^{2} \alpha\right]^{1 / 2}$

for an oblate spheroid. The superscripts TM and TE are related to two cases of the polarization of incident radiation (TM and TE modes).

The efficiency factors $Q_{\mathrm{pr} 1,2}^{\mathrm{TM}, \mathrm{TE}}$ are calculated from the solution to the light scattering problem for spheroids (see Voshchinnikov \& Farafonov 1993 for details). For both modes, they can be expressed as follows:

$$
\begin{gathered}
Q_{\mathrm{pr} 1}\left(m_{\lambda}, x_{V}, a / b, \alpha\right)=Q_{\mathrm{ext}}\left(m_{\lambda}, x_{V}, a / b, \alpha\right)- \\
\mathcal{A}\left(m_{\lambda}, x_{V}, a / b, \alpha\right) \cos \alpha-\mathcal{B}\left(m_{\lambda}, x_{V}, a / b, \alpha\right) \sin \alpha,
\end{gathered}
$$

$$
\begin{array}{r}
Q_{\mathrm{pr} 2}\left(m_{\lambda}, x_{V}, a / b, \alpha\right)= \\
\mathcal{A}\left(m_{\lambda}, x_{V}, a / b, \alpha\right) \sin \alpha-\mathcal{B}\left(m_{\lambda}, x_{V}, a / b, \alpha\right) \cos \alpha,
\end{array}
$$

where $Q_{\text {ext }}=Q_{\text {sca }}+Q_{\text {abs }}$ is the extinction efficiency factor, and the coefficients $\mathcal{A}$ and $\mathcal{B}$ are

$$
\begin{aligned}
& \mathcal{A}=\mathcal{K}\left(x_{V}, a / b, \alpha\right) \int_{0}^{2 \pi} \int_{0}^{\pi} i(\theta, \varphi) \cos \theta \sin \theta \mathrm{d} \theta \mathrm{d} \varphi \\
& \mathcal{B}=\mathcal{K}\left(x_{V}, a / b, \alpha\right) \int_{0}^{2 \pi} \int_{0}^{\pi} i(\theta, \varphi) \sin ^{2} \theta \cos \varphi \mathrm{d} \theta \mathrm{d} \varphi .
\end{aligned}
$$

In Eqs. $(11)-(12), \mathcal{K}$ is a parameter, $i(\theta, \varphi)$ the dimensionless intensity of scattered radiation (phase function; Fig. 1). From a symmetry consideration, it is clear that for spheroids $Q_{\mathrm{pr} 2}=0$ if $\alpha=0^{\circ}$ or $90^{\circ}$. The detailed expressions for calculations of the factors $Q_{\mathrm{pr} 1,2}$ are given in the paper of Voshchinnikov (1990).

\subsubsection{Rotating grains}

For randomly aligned particles, the Planck mean crosssections have to be averaged over all orientations

$\overline{\bar{C}}_{\mathrm{pr} 1}^{3 \mathrm{D}}=\int_{0}^{\pi / 2} \frac{1}{2}\left[\bar{Q}_{\mathrm{pr} 1}^{\mathrm{TM}}(\alpha)+\bar{Q}_{\mathrm{pr} 1}^{\mathrm{TE}}(\alpha)\right] G(\alpha) \sin \alpha \mathrm{d} \alpha$,

$\overline{\bar{C}}_{\mathrm{pr} 2}^{3 \mathrm{D}}=0$.

For perfect dynamical alignment, the Planck mean cross-sections are averaged over all rotational angles $\phi$. This gives for prolate spheroids

$\overline{\bar{C}}_{\mathrm{pr} 1}^{2 \mathrm{D}}(\Omega)=\frac{2}{\pi} \int_{0}^{\pi / 2} \frac{1}{2}\left[\bar{Q}_{\mathrm{pr} 1}^{\mathrm{TM}}(\alpha)+\bar{Q}_{\mathrm{pr} 1}^{\mathrm{TE}}(\alpha)\right] G(\alpha) \mathrm{d} \phi$,

$$
\begin{array}{r}
\overline{\bar{C}}_{\mathrm{pr} 2}^{2 \mathrm{D}}(\Omega)=\frac{2}{\pi} \int_{0}^{\pi / 2} \frac{1}{2}\left[\bar{Q}_{\mathrm{pr} 2}^{\mathrm{TM}}(\alpha)+\bar{Q}_{\mathrm{pr} 2}^{\mathrm{TE}}(\alpha)\right] \times \\
G(\alpha) \csc \alpha \cos \Omega \cos \phi \mathrm{d} \phi
\end{array}
$$

where the angle $\alpha$ is connected with $\Omega$ and $\phi(\cos \alpha=$ $\sin \Omega \cos \phi$ ). For oblate spheroids randomly aligned in a plane, we have $\Omega=\alpha$ and

$\overline{\bar{C}}_{\mathrm{pr} 1,2}^{2 \mathrm{D}}(\Omega)=\frac{1}{2}\left[\bar{Q}_{\mathrm{pr} 1,2}^{\mathrm{TM}}(\Omega)+\bar{Q}_{\mathrm{pr} 1,2}^{\mathrm{TE}}(\Omega)\right] G(\Omega)$.

\subsection{Equation of motion and drift velocity}

The radiation pressure force $\mathbf{F}_{\mathrm{pr}}$ is the main force acting on dust grains in the envelopes of late-type giants. Its ratio to the gravitational force $\beta \equiv F_{\mathrm{pr}} / F_{\mathrm{g}}$ is much larger than 1 in almost all cases (Voshchinnikov \& Il'in 1983b). As weak magnetic fields and small electric charge on grains are expected in the envelopes, we can neglect the Coulomb drag and Lorentz forces (see Il'in 1994 for details). Thus, the equation of the stationary grain motion should be

$\mathbf{F}_{\mathrm{pr}}+\mathbf{F}_{\mathrm{coll}}=0$,

where $\mathbf{F}_{\text {coll }}$ is the drag force due to collisions of a grain with surrounding gas particles. It is proportional to the gas density, the grain velocity relative to the gas $\mathbf{v}_{\text {drift }}=$ $\mathbf{v}_{\mathrm{d}}-\mathbf{v}_{\text {gas }}$, and the geometrical cross-section of the grain. For rotating grains, the averaged geometrical cross-section $\bar{G}^{3 \mathrm{D}}$ or $\bar{G}(\Omega)$ should be used. The corresponding expressions are given in Appendix A.

The force $\mathbf{F}_{\text {coll }}$ is antiparallel to $\mathbf{v}_{\text {drift }}$ for spherical particles. For non-spherical grains and subsonic drift velocity, the angle between these two vectors depends on the type of reflection of the gas particles from the grain surface. If the mirror reflection occurs, then $\mathbf{F}_{\text {coll }}$ is perpendicular to the major semiaxis of a spheroidal grain; for diffuse reflection, $\mathbf{F}_{\text {coll }}$ forms a small angle with $-\mathbf{v}_{\text {drift }}$, and the value of this angle depends on the difference between the gas and dust temperatures. The angle should be close to zero for any type of reflection in the case of supersonic drift velocity.

From the equation $F_{\mathrm{pr} 1}=F_{\text {coll }}$, the mean drift velocity of a rotating grain is

$$
\begin{array}{r}
v_{\text {drift }}^{\text {spheroid }}=\frac{L_{\star}}{\dot{M} c} \frac{v_{\text {gas }}}{\bar{w}} \frac{\overline{\bar{C}}_{\mathrm{pr} 1}}{\bar{G} \sqrt{\pi} f_{w}}, \\
f_{w}=\frac{8}{3 \pi}+\frac{1}{3} \sqrt{\frac{T_{\text {dust }}}{T_{\text {gas }}}} \\
\bar{w} \approx w_{\mathrm{H}}\left(X_{\mathrm{H}}+\frac{X_{\mathrm{H}_{2}}}{\sqrt{2}}+\frac{Y}{2}+\frac{Z}{4}\right)
\end{array}
$$

for subsonic motion and

$$
v_{\text {drift }}^{\text {spheroid }}=\left(\frac{L_{\star}}{\dot{M} c} v_{\text {gas }} \frac{\overline{\bar{C}}_{\mathrm{pr} 1}}{\bar{G}}\right)^{1 / 2}
$$


for supersonic motion. Here, $L_{\star}$ is the stellar luminosity, $\dot{M}$ the mass loss rate, $\bar{w}$ the sound velocity, $X_{\mathrm{H}}, X_{\mathrm{H}_{2}}, Y, Z$ are the relative abundances (by mass) of hydrogen (atoms and molecules), helium and heavy elements, respectively.

Thus, the ratio of the drift velocities for spheroidal and spherical particles of the same volume is determined only by the cross-sections of the particles

$\frac{v_{\mathrm{drift}}^{\text {spheid }}}{v_{\mathrm{drift}}^{\text {sphere }}}=\left(\frac{\overline{\bar{C}}_{\mathrm{pr} 1}}{\bar{G}} \frac{\pi r_{V}^{2}}{C_{\mathrm{pr}}}\right)^{1 / n}$,

where $n=1$ for subsonic motion and $n=2$ for supersonic motion.

\section{Results and discussion}

The Planck mean cross-sections $\bar{C}_{\mathrm{pr} 1,2}$ for various prolate and oblate spheroids and spheres of different sizes and chemical compositions have been calculated. Then the cross-sections were averaged over the grain orientations and both the radiation pressure force and the dust drift velocities were estimated.

The radial component of the radiation pressure force $F_{\text {pr1 }}$ determines the dust drift velocity and as a result the radiation momentum transfer to the gas and the radial gas outflow velocity. To evaluate the grain shape effects we calculated the ratios of the forces $F_{\mathrm{pr} 1} / F_{\mathrm{pr}}$ and of the velocities $v_{\mathrm{drift}}^{\text {spheroid }} / v_{\mathrm{drift}}^{\text {sphere }}$ for spheroidal and spherical particles of the same volume. The Planck mean factors for spheres presented in tables can be used for estimations of the absolute values of drift velocities. To illustrate its relative strength the transversal component of the radiation pressure force $F_{\mathrm{pr} 2}$ is compared with the radial one $F_{\mathrm{pr} 1}$.

\subsection{Radial radiation pressure}

The values of $F_{\mathrm{pr} 1} / F_{\mathrm{pr}}$ and $v_{\mathrm{drift}}^{\text {spheroid }} / v_{\mathrm{drift}}^{\text {sphere }}$ for subsonic motion $(n=1$ in Eq. (21)) are given in Table 1 for the particles consisting of amorphous carbon and astronomical silicate. The data are presented for two cases of dynamically aligned grains $\left(\Omega=0^{\circ}\right.$ and $\left.90^{\circ}\right)$, the ratios for other angles $\Omega$ lie between these values. For $3 \mathrm{D}$-orientation, the approximation

$\overline{\bar{F}}_{\mathrm{pr} 1}^{3 \mathrm{D}} \approx \frac{1}{3} \overline{\bar{F}}_{\mathrm{pr} 1}^{2 \mathrm{D}}\left(\Omega=0^{\circ}\right)+\frac{2}{3} \overline{\bar{F}}_{\mathrm{pr} 1}^{2 \mathrm{D}}\left(\Omega=90^{\circ}\right)$

works sufficiently well and the results obtained are not shown in Table 1.

As follows from Table 1 and Fig. 2 , the ratio $F_{\mathrm{pr} 1} / F_{\mathrm{pr}}$ usually grows with the increase of the aspect ratio $a / b$ and the absorptive capacity of the grain material. A very significant effect is found for small metallic spheroids (Fig. 2). It was firstly mentioned by Il'in (1994) and can be explained by extremely large absorption of radiation with the electric vector oscillating along the major axis of a particle (TM mode for a prolate spheroid and TE mode

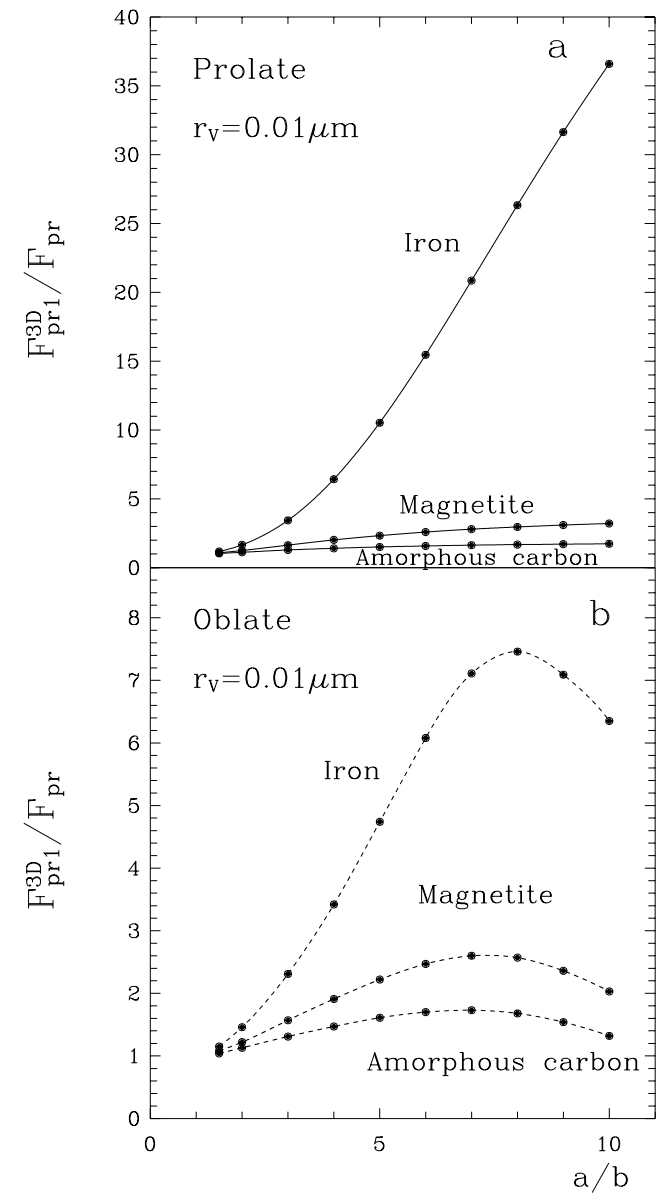

Fig. 2. The shape dependence of the ratio of radiation pressure forces for prolate (solid curves; a)) and oblate (dashed curves; b)) spheroids with $3 \mathrm{D}$-orientation and spheres. The composition of absorbing materials is indicated

for a oblate one). This fact is well known in the theory of electromagnetic radiation scattering (van de Hulst 1957) and is related to different behaviour of surface waves for conductors. It is obvious that the maximum effect occurs when the major semiaxis of a particle is perpendicular to the wave-vector, i.e. in the case of $2 \mathrm{D}$-orientation and $\Omega=0^{\circ}$.

If we consider the silicate grains, the ratio $F_{\mathrm{pr} 1} / F_{\mathrm{pr}}$ reaches a maximum with the growth of the particle size $\left(r_{V} \approx 0.1 \mu \mathrm{m}\right.$ if $\left.T_{\star}=2500 \mathrm{~K}\right)$. After the maximum, the ratio $F_{\text {pr1 }} / F_{\text {pr }}$ becomes below 1 , if the major axis of a rotating grain is not perpendicular to the wave-vector (i.e. $\left.\Omega>0^{\circ}\right)$. This is connected with the growing role of the scattered radiation that leads to an increase of the angle between $\mathbf{F}_{\mathrm{pr}}$ and $\mathbf{k}$ (see Fig. 1). Therefore, the projection of $\mathbf{F}_{\mathrm{pr}}$ on the radial direction decreases.

The common feature of the grain motion is that spheroids with size $r_{V} \lesssim 0.1 \mu \mathrm{m}$ are blown slightly faster than equivolume spheres. For strongly absorbing grains the effect can be extremely large. For instance, the drift 
Table 1. The ratios of the radiation pressure force and the drift velocity for spheroids and spheres having an equal volume

\begin{tabular}{|c|c|c|c|c|c|c|c|c|c|c|}
\hline \multirow[b]{3}{*}{$a / b$} & \multirow[b]{3}{*}{$r_{V}, \mu \mathrm{m}$} & \multicolumn{4}{|c|}{ Prolate spheroid } & \multicolumn{4}{|c|}{ Oblate spheroid } & \multirow{3}{*}{$\begin{array}{c}\text { Sphere } \\
\bar{Q}_{\mathrm{pr}}\end{array}$} \\
\hline & & \multicolumn{2}{|c|}{$F_{\mathrm{pr} 1}^{2 \mathrm{D}} / F_{\mathrm{pr}}$} & \multicolumn{2}{|c|}{$v_{\text {drift }}^{\text {spheroid }} / v_{\text {drift }}^{\text {sphere }}$} & \multicolumn{2}{|c|}{$F_{\mathrm{pr} 1}^{2 \mathrm{D}} / F_{\mathrm{pr}}$} & \multicolumn{2}{|c|}{$v_{\mathrm{drift}}^{\text {spheroid }} / v_{\mathrm{drift}}^{\text {sphere }}$} & \\
\hline & & $\Omega=0^{\circ}$ & $90^{\circ}$ & $0^{\circ}$ & $90^{\circ}$ & $0^{\circ}$ & $90^{\circ}$ & $0^{\circ}$ & $90^{\circ}$ & \\
\hline \multicolumn{11}{|c|}{ Amorphous carbon } \\
\hline \multirow[t]{6}{*}{2.0} & 0.01 & 1.29 & 1.04 & 1.03 & 1.07 & 1.40 & 0.99 & 0.88 & 1.25 & 0.012 \\
\hline & 0.05 & 1.30 & 1.04 & 1.03 & 1.07 & 1.40 & 0.99 & 0.88 & 1.25 & 0.071 \\
\hline & 0.10 & 1.29 & 1.01 & 1.02 & 1.04 & 1.39 & 0.97 & 0.87 & 1.22 & 0.23 \\
\hline & 0.20 & 1.13 & 0.97 & 0.90 & 1.00 & 1.20 & 0.96 & 0.76 & 1.21 & 0.89 \\
\hline & 0.30 & 1.06 & 0.98 & 0.84 & 1.01 & 1.10 & 0.98 & 0.69 & 1.23 & 1.48 \\
\hline & 0.50 & 1.06 & 0.98 & 0.84 & 1.00 & 1.09 & 0.95 & 0.69 & 1.20 & 1.93 \\
\hline \multirow[t]{6}{*}{4.0} & 0.01 & 1.78 & 1.23 & 1.12 & 1.14 & 2.01 & 1.20 & 0.80 & 1.90 & \\
\hline & 0.05 & 1.78 & 1.21 & 1.12 & 1.12 & 1.99 & 1.19 & 0.79 & 1.89 & \\
\hline & 0.10 & 1.70 & 1.09 & 1.07 & 1.01 & 1.91 & 1.09 & 0.76 & 1.72 & \\
\hline & 0.20 & 1.31 & 0.95 & 0.82 & 0.88 & 1.48 & 0.95 & 0.59 & 1.51 & \\
\hline & 0.30 & 1.16 & 0.96 & 0.73 & 0.89 & 1.25 & 0.95 & 0.49 & 1.51 & \\
\hline & 0.50 & 1.16 & 1.00 & 0.73 & 0.93 & 1.17 & 0.97 & 0.46 & 1.53 & \\
\hline \multicolumn{11}{|c|}{ Astronomical silicate } \\
\hline \multirow[t]{6}{*}{2.0} & 0.01 & 1.16 & 1.00 & 0.92 & 1.03 & 1.26 & 0.97 & 0.79 & 1.22 & 0.0025 \\
\hline & 0.05 & 1.17 & 0.99 & 0.93 & 1.02 & 1.27 & 0.96 & 0.80 & 1.21 & 0.016 \\
\hline & 0.10 & 1.23 & 0.95 & 0.97 & 0.98 & 1.37 & 0.90 & 0.86 & 1.14 & 0.064 \\
\hline & 0.20 & 1.15 & 0.93 & 0.91 & 0.95 & 1.30 & 0.88 & 0.82 & 1.11 & 0.31 \\
\hline & 0.30 & 1.03 & 0.94 & 0.82 & 0.96 & 1.10 & 0.92 & 0.69 & 1.16 & 0.63 \\
\hline & 0.50 & 0.99 & 0.95 & 0.78 & 0.98 & 0.96 & 0.95 & 0.61 & 1.20 & 1.06 \\
\hline \multirow[t]{6}{*}{4.0} & 0.01 & 1.37 & 1.07 & 0.86 & 0.98 & 1.59 & 1.04 & 0.63 & 1.65 & \\
\hline & 0.05 & 1.38 & 1.04 & 0.87 & 0.96 & 1.60 & 1.02 & 0.64 & 1.62 & \\
\hline & 0.10 & 1.42 & 0.91 & 0.89 & 0.84 & 1.70 & 0.87 & 0.68 & 1.38 & \\
\hline & 0.20 & 1.26 & 0.82 & 0.80 & 0.76 & 1.56 & 0.76 & 0.62 & 1.21 & \\
\hline & 0.30 & 1.07 & 0.82 & 0.67 & 0.76 & 1.22 & 0.78 & 0.48 & 1.24 & \\
\hline & 0.50 & 0.98 & 0.86 & 0.62 & 0.79 & 0.90 & 0.84 & 0.36 & 1.33 & \\
\hline
\end{tabular}

velocity of even nonaligned iron spheroids and spheres may differ in 5-10 or more times.

For large aligned particles, the difference in the final velocities of spheres and spheroids mainly depends on the ratio of the average geometrical cross-sections (see Appendix A). However, a difference in radiation pressure is present as well. For example, for oblate particles, the ratio of their velocities (see two last columns of Table 1 ) is not equal to $\bar{G}\left(\Omega=0^{\circ}\right) / \bar{G}\left(\Omega=90^{\circ}\right)=a / b$ as it should be without this influence.

It should be also noted that in the case of $2 \mathrm{D}$ orientation the grain shape effects are more pronounced for oblate particles in comparison with prolate ones (if we compare the particles of the same volume with $a / b \lesssim$ $5-7)$. They enlarge with the growth of the aspect ratio $a / b$ and become maximal for needle-like particles.

\subsection{Transversal radiation pressure}

The appearance of the transversal component of the radiation pressure force $F_{\mathrm{pr} 2}$ is explained by the azimuthal asymmetry of the radiation recoil for a non-spherical particle. The strength of this component is tightly connected with the particle orientation relative to the wave-vector of incident radiation and also strongly depends on the aspect ratio, size and composition of grains. It should be noted that we study the scattered radiation in a Cartesian coordinate system in which the $z$-axis coincides with the rotation axis of a spheroid. For oblate particles, this leads to the change of the direction of $F_{\mathrm{pr} 2}$ in Fig. 1. Formally, it means that the second term in Eq. (10) becomes larger than the first one, i.e. $Q_{\mathrm{pr} 2}<0$.

The transversal component can be very large and reaches up to $30 \%$ or more of the radial one in the cases considered (Table 2, Fig. 3).

The growth of the ratio $F_{\mathrm{pr} 2} / F_{\mathrm{pr} 1}$ with the particle size reflects the increasing azimuthal asymmetry of phase function and particle's albedo. After a peak value (near $\left.r_{V} \approx 0.3 \mu \mathrm{m}\right)$, the ratio $F_{\mathrm{pr} 2} / F_{\mathrm{pr} 1}$ slightly decreases because of more symmetric azimuthal scattering of radiation by larger particles. 
Table 2. The ratio of the transversal and radial components of the radiation pressure force $F_{\mathrm{pr} 2}^{2 \mathrm{D}} / F_{\mathrm{pr} 1}^{2 \mathrm{D}}$ for dynamically aligned spheroidal grains, $\Omega=45^{\circ}$

\begin{tabular}{|c|c|c|c|c|c|c|c|c|}
\hline \multirow[b]{2}{*}{$r_{V}, \mu \mathrm{m}$} & \multicolumn{4}{|c|}{ Prolate spheroid } & \multicolumn{4}{|c|}{ Oblate spheroid } \\
\hline & $a / b=1.1$ & 1.5 & 2.0 & 4.0 & 1.1 & 1.5 & 2.0 & 4.0 \\
\hline \multicolumn{9}{|c|}{ Amorphous carbon } \\
\hline 0.05 & $0.01 \%$ & $0.03 \%$ & $0.06 \%$ & $0.13 \%$ & $-0.01 \%$ & $-0.06 \%$ & $-0.11 \%$ & $-0.22 \%$ \\
\hline 0.10 & 0.15 & 0.51 & 0.83 & 1.61 & -0.19 & -1.05 & -1.85 & -3.29 \\
\hline 0.20 & 0.54 & 1.97 & 3.31 & 6.35 & -0.62 & -3.48 & -6.31 & -12.1 \\
\hline 0.30 & 0.64 & 2.45 & 4.22 & 8.51 & -0.78 & -3.90 & -6.82 & -13.9 \\
\hline 0.50 & 0.37 & 1.84 & 3.52 & 8.13 & -0.58 & -2.23 & -3.67 & -9.20 \\
\hline \multicolumn{9}{|c|}{ Astronomical silicate } \\
\hline 0.05 & $0.02 \%$ & $0.09 \%$ & $0.16 \%$ & $0.39 \%$ & $-0.03 \%$ & $-0.15 \%$ & $-0.25 \%$ & $-0.49 \%$ \\
\hline 0.10 & 0.33 & 1.16 & 1.90 & 3.75 & -0.39 & -2.04 & -3.44 & -6.12 \\
\hline 0.20 & 1.37 & 5.01 & 8.07 & 13.3 & -1.73 & -8.59 & -14.3 & -23.7 \\
\hline 0.30 & 1.84 & 7.33 & 12.1 & 19.2 & -2.74 & -12.0 & -19.4 & -32.3 \\
\hline 0.50 & 1.31 & 7.38 & 13.0 & 20.8 & -2.76 & -10.9 & -18.2 & -28.6 \\
\hline
\end{tabular}

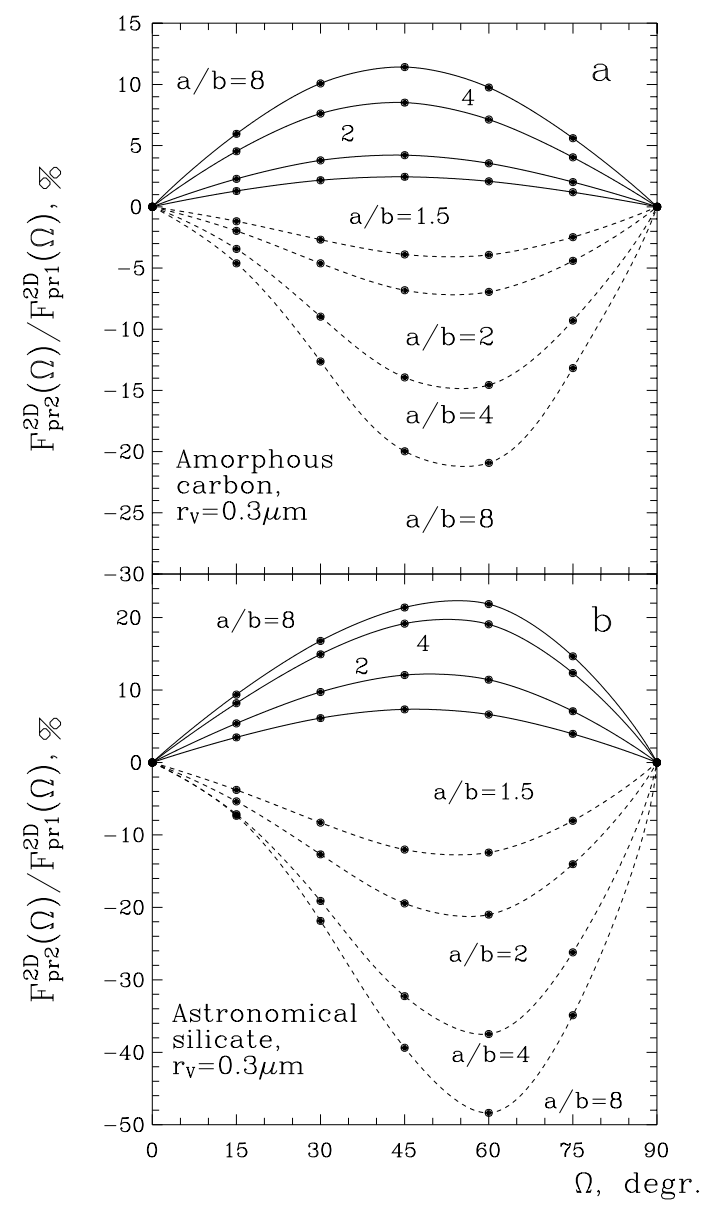

Fig. 3. The angular dependence of the transversal component of the radiation pressure force for dynamically aligned prolate (solid curves) and oblate (dashed curves) spheroids consisting of amorphous carbon a) and astronomical silicate $\mathbf{b}$ ). The values of the aspect ratio $a / b$ are indicated
As follows from Fig. 3, the values of $\Omega$ at which the ratio $F_{\mathrm{pr} 2} / F_{\mathrm{pr} 1}$ reaches a maximum depend on the particle size and are in the interval $45^{\circ}-60^{\circ}$. Note that if the particle shape is not axially symmetric particles, the angular dependence of the transversal component and its strength may differ significantly from that presented in Fig. 3.

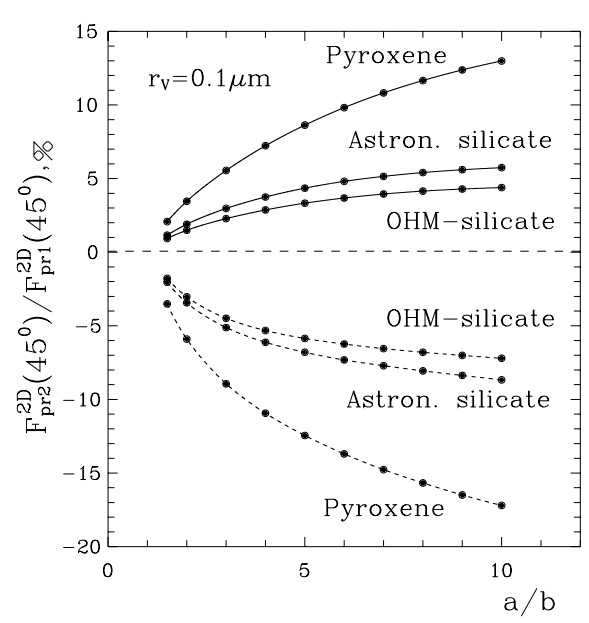

Fig. 4. The shape dependence of the transversal component of the radiation pressure force for dynamically aligned $\left(\Omega=45^{\circ}\right)$ prolate (solid curves) and oblate (dashed curves) spheroids. The composition of silicate material is indicated

The higher absorptive capacity (or dirtiness) of silicates leads to the smaller ratios of $F_{\mathrm{pr} 2} / F_{\mathrm{pr} 1}$. This fact is illustrated by Fig. 4, where the distinction between three silicate species is mainly related to the difference in particle's albedo. 
The role of the transversal component of the radiation pressure force may be as follows. First, as the force is not parallel to the incident radiation wave-vector (and hence the radius-vector), the more is $F_{\mathrm{pr} 2} / F_{\mathrm{pr} 1}$, the less is the radial drift velocity of grains. Second, the path length of a non-spherical dust grain through the shell (and therefore the number of collisions with gas particles) should be larger in comparison with the spherical particle of the same volume. Third, provided there is a preferable stable orientation of grains, a non-radial outflow of gas may be initiated.

\subsection{Porous grains}

With the growth of the fraction of vacuum $f$ the grain porosity increases and the effective refraction index of a particle reduces. This leads to a decrease of the ratios $F_{\mathrm{pr} 1} / F_{\mathrm{pr}}$ and $v_{\mathrm{drift}}^{\text {spheroid }} / v_{\mathrm{drift}}^{\text {sphere }}$ for metallic grains as the radiation pressure is mainly determined by absorption.

For silicate particles, the situation is more complicated: both the radial and transversal components of the radiation pressure force can decrease with increasing porosity of circumstellar grains as it is seen from Table 3. But the effect appears only for aggregates containing more than $\sim 30 \%$ of vacuum.

Table 3. The behaviour of radiation pressure force for porous prolate spheroidal grains consisting of astronomical silicate, 2D-orientation, $r_{V}=0.3 \mu \mathrm{m}, a / b=4$

\begin{tabular}{ccccc}
\hline & \multicolumn{2}{c}{$F_{\mathrm{pr} 1}^{2 \mathrm{D}} / F_{\mathrm{pr}}$} & $F_{\mathrm{pr} 2}^{2 \mathrm{D}} / F_{\mathrm{pr} 1}^{2 \mathrm{D}}$ & Sphere \\
\cline { 2 - 4 } $1-f$ & $\Omega=0^{\circ}$ & $90^{\circ}$ & $45^{\circ}$ & $\bar{Q}_{\mathrm{pr}}$ \\
\hline 1.0 & 1.07 & 0.82 & $19.2 \%$ & 0.63 \\
0.9 & 1.07 & 0.82 & 19.3 & 0.52 \\
0.7 & 1.07 & 0.83 & 19.2 & 0.33 \\
0.5 & 1.07 & 0.84 & 18.1 & 0.18 \\
0.3 & 1.07 & 0.86 & 15.7 & 0.07 \\
0.1 & 1.04 & 0.91 & 8.63 & 0.012 \\
\hline
\end{tabular}

It should be also mentioned that the approach used (Sect. 2.2.4) implies the uniform distribution of vacuum in a particle. But if a grain has one or several bubbles, the optical properties of such hollow particle may strongly differ from those of both the porous and compact particles of the same volume. The radiation pressure on a hollow dielectric particle may exceed that on a compact one because of the total internal reflection. Such an effect for infinite cylinders has been demonstrated by Kokody (1989).

\subsection{Effects of stellar temperature variations and polarization}

A decrease of the stellar temperature is usually accompanied with an increase of the radial component of the radiation pressure force acting on a non-spherical particle. This fact is illustrated by Fig. 5, where the ratio of the drift velocities is plotted. Note that the values given for the effective temperatures as low as $500 \mathrm{~K}$ are related to the possible conditions in the outer layers of the optically thick envelopes. It is seen that the distinction is not very large and is more emphasized for carbon grains and larger values of the aspect ratio. The ratio $v_{\mathrm{drift}}^{\text {spheroid }} / v_{\mathrm{drift}}^{\text {sphere }}$ is independent of $T_{\star}$ when the grain size $r_{V}<0.03 \mu \mathrm{m}$.

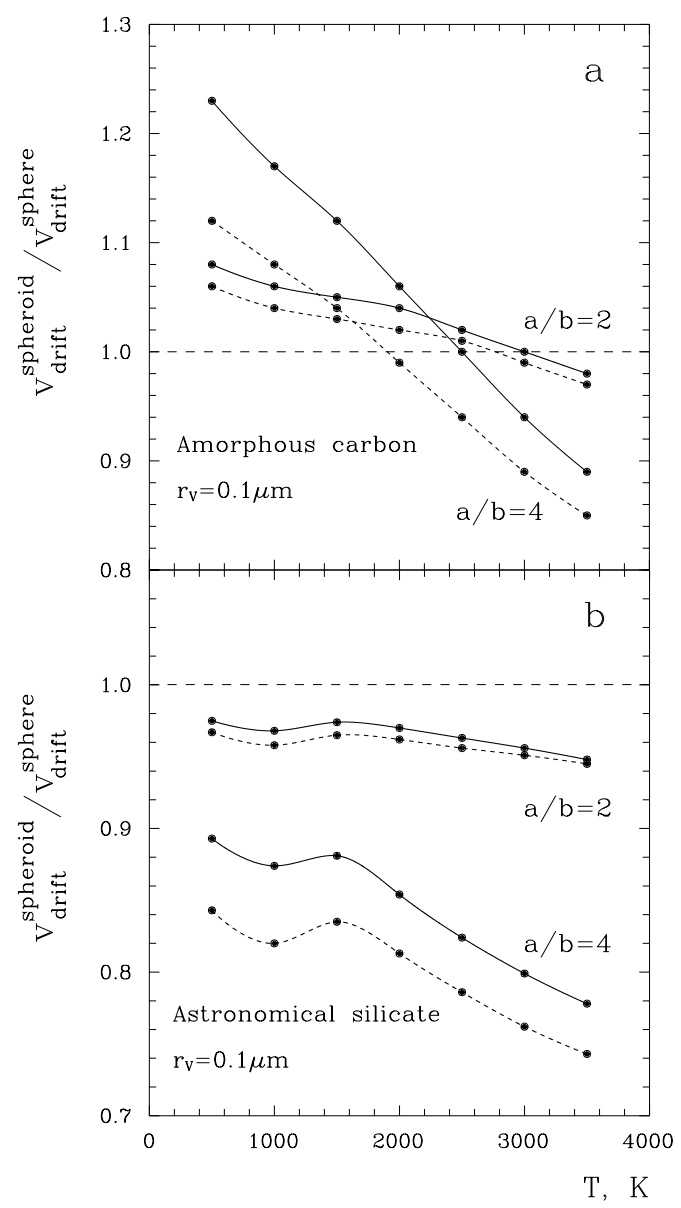

Fig. 5. The temperature dependence of the drift velocity for prolate (solid curves) and oblate (dashed curves) spheroids from amorphous carbon a) and astronomical silicate b) in the case of $3 \mathrm{D}$-orientation. The values of the aspect ratio $a / b$ are indicated

Any increase of the stellar temperature reflects in a rise of the transversal component (Fig. 6). This is due to the growth of the effective size parameter of the particle $x_{\text {eff }}=2 \pi r_{V} / \lambda_{\text {eff }}$ for hotter stars and hence the increase of the role of scattering. 


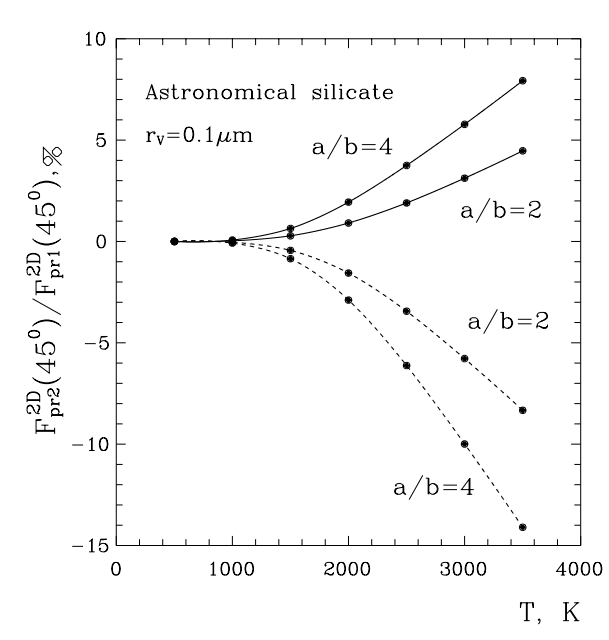

Fig. 6. The temperature dependence of the transversal component of the radiation pressure force for dynamically aligned $\left(\Omega=45^{\circ}\right)$ prolate (solid curves) and oblate (dashed curves) spheroids. The values of the aspect ratio $a / b$ are indicated

Since many late-type giants possess brightness variations, the common feature should be an increase of the radial component and a decrease of the transversal one during brightness minima in comparison with maxima.

Aligned non-spherical grains in the inner parts of the envelopes can polarize stellar radiation. In principle, the local polarization might be rather large. Then the polarized radiation incident on grains in the outer parts can to strengthen or weaken the shape effects discussed above.

\subsection{Optically thick shells}

Equation (4) used above to evaluate the radiation pressure force is strictly applicable only for optically thin shells. In the case of large optical thickness, one should insert into it the integration over all solid angles $\omega$ and take into account the angular dependence of the flux $H_{\nu}(\omega)$ that might be obtained from radiative transfer modelling.

When the anisotropy of the flux is not pronounced both components of the radiation pressure force should be limited. On the other hand, even if $H_{\nu}(\omega)$ has a sharp maximum but mainly includes thermal radiation of dust, the transversal component should be small because of low temperature of the radiation source (Fig. 6).

For small particles, the shape effect - the difference of the radiation pressure forces acting on a spheroid and a equivolume sphere should be essential also in the case of optically thick shells as the effect is weakly affected by the source temperature (see Fig. 5). Thus, if $H_{\nu}(\omega)$ has an anisotropy, radiation pressure will occur and the shape effect will depend on the orientation of non-spherical particles relative to the direction of maximum flux.

\section{Conclusions}

We have calculated the radiation pressure force acting on prolate and oblate spheroidal grains of different size, aspect ratio, chemical composition and alignment. The consideration has been done for conditions typical of the envelopes of carbon-rich or oxygen-rich giants. The force and the grain drift velocity (relative to gas) obtained as a solution of simple equation of dust motion have been compared for spheroids and spheres of the same volume.

It is found that for spheroids with radii of equivolume sphere $r_{V} \lesssim 0.1 \mu \mathrm{m}$ the radiation pressure force commonly is greater than that for spheres. The effect is in particular strong for highly absorbing particles with $r_{V} \lesssim 0.03-0.05 \mu \mathrm{m}$. It is explained by the resonance absorption of incident radiation whose electric vector is parallel to the major axis of a particle. As a result the velocity of a sphere and equivolume spheroids consisting of iron can differ in 5-10 times or more.

Another effect is the existence of the component of the radiation pressure force perpendicular to the wave-vector in the case of oblique incidence of radiation. It is caused by an azimuthal asymmetry of geometry of light scattering by non-spherical particles. The transversal component of the force is prominent for dielectric particles and can reach up to $30-50 \%$ of the radial one for silicate grains of the size $r_{V} \approx 0.3 \mu \mathrm{m}$. This component should increase the path of non-spherical grains and the number of their collisions with the gas particles in the envelopes of late-type stars. Such an effect can enlarge the living time of silicate grains in the envelopes that may facilitate the process of crystallization of amorphous silicates.

The grain porosity generally decreases both radial and transversal components of the radiation pressure force if the particles of the same volume are considered. For silicate grains, this reduction appears for aggregates containing more than $\sim 30 \%$ of vacuum.

Variations of the effective temperature of the stars can lead to a strengthening of the radial component of the radiation pressure force during brightness minima and of the transversal one during maxima.

Acknowledgements. The authors are thankful to R. Stognienko for comments. V.B.I. acknowledges the Alexander von Humboldt Foundation for the financial support. The work was partly supported by the Committee of High Education for Russian Federation (grant 95-0-3.1-1).

\section{A. Average geometrical cross-sections}

The expressions for the geometrical cross-sections of rotating spheroids can be obtained if we average the crosssections $G(\alpha)$ (Eqs. (7) - (8)) according to the equations similar to Eqs. (13), (15). For the 3D-orientation, we obtain $(e=a / b)$

$\bar{G}^{3 \mathrm{D}}=\pi r_{V}^{2}\left[\frac{1}{e}+\frac{\arcsin \left(\sqrt{e^{2}-1} / e\right)}{\sqrt{e^{2}-1} / e}\right] \frac{\sqrt[3]{e}}{2}$ 
for prolate spheroids and

$\bar{G}^{3 \mathrm{D}}=\pi r_{V}^{2}\left[e+\frac{\ln \left(e+\sqrt{e^{2}-1}\right)}{\sqrt{e^{2}-1}}\right] \frac{1}{2 \sqrt[3]{e}}$

for oblate spheroids. For the perfect rotational orientation, the cross-sections are

$\bar{G}^{2 \mathrm{D}}(\Omega)=\pi r_{V}^{2} \frac{2}{\pi} E\left(\frac{e^{2}-1}{e^{2}} \sin ^{2} \Omega\right) \sqrt[3]{e}$

for prolate spheroids and

$\bar{G}^{2 \mathrm{D}}(\Omega)=G(\Omega)$

for oblate ones. Here, $E(m)$ is the complete elliptic integral of the second kind.

\section{References}

Bagnulo S., Doyle J.G., Griffin I.P., 1995, A\&A 301, 501

Baron Y., de Muizon M., Papoular R., Pégourié B., 1987, A\&A 186,271

Bohren C.F., Huffman D.R., 1983, Absorption and scattering of light by small particles. J. Wiley \& Sons, New York

Cadwell B.J., Wang H., Feigelson E.D., Frenklach M., 1994, ApJ 429, 285

David P., Pégourié B., 1995, A\&A 293, 833

Dolginov A.Z., Gnedin Yu.N., Silant'ev N.A., 1979, Propagation and polarization of radiation in cosmic medium, Nauka, Moscow

Dorschner J., Begemann B., Henning Th., Jäger C., Mutschke H., 1995, A\&A 300, 503

Draine B.T., 1981, in: Iben I., Renzini A. (eds.), Physical Processes in Red Giants. Dordrecht, Reidel, p. 317

Dyck H.M., Jennings M.C., 1971, AJ 76, 431

Fadeyev Yu. A., 1987, in: I. Appenzeller, C. Jordan (eds.), Circumstellar Matter, IAU Symp. 122. Dordrecht, Reidel, p. 515

Fleischer A.J., Gauger A., Sedlmayr E., 1992, A\&A 266, 321

Gail H.-P., Sedlmayr E., 1984, A\&A 132, 163

Gail H.-P., Sedlmayr E., 1985, A\&A 148, 183

Goebel J.H., Moseley H., 1985, ApJ 290, L35
Habing H.J., Tignon J., Tielens A.G.G.M., 1994, A\&A 286, 523

Huffman D.R., Stapp J.L., 1973, in: Greenberg J.M., van de Hulst H.C. (eds.), Interstellar dust and related topics, IAU Symp. 52. Dordrecht, Dordrecht, p. 297

Il'in V.B., 1994, A\&A 281, 486

Jäger C., Mutschke H., Begemann B., Dorschner J., Henning Th., 1994, A\&A 292, 641

Jones T.W., Merrill K.M., 1976, ApJ 209, 509

Jura M., 1994, ApJ 434, 713

Jura M., 1996, ApJ 472, 806

Kerker M., 1981, Planet. Space Sci. 29, 127

Kokody N.G., 1989, Opt. Spectrosc. 66, 1089

Lafon J.-P.J., Berruyer N., 1991, A\&AR 2, 249

Laor A., Draine B.T., 1993, ApJ 402, 441

Little-Marenin I., 1986, ApJ 307, L15

Lorenz-Martins S., Lefèvre J., 1994, A\&A 291, 831

Mukai T., Ishimoto H., Kozasa T., Blum J., Greenberg J.M., 1992, A\&A 262, 315

Netzer N., Elitzur M., 1993, ApJ 410, 701

Ordal M.A., Bell R.J., Alexander R.W., Newquist L.A., Querry M.R., 1988, Appl. Opt. 27, 1203

Ossenkopf V., Henning Th., Mathis J.S., 1992, A\&A 261, 567

Pégourié B., 1987, Ap\&SS 136, 133

Pollack J.B., Hollenbach D., Beckwith S., Simonelli D.P., Roush T., Fong W., 1994, ApJ 421, 615

Rouleau F., Martin P.G., 1991, ApJ 377, 526

Shawl S.J., 1975, AJ 80, 602

Spitzer L., 1978, Physical Processes in the Interstellar Medium. J. Wiley \& Sons, New York

Steyer T.R., 1974, PhD Thesis, Univ. Arizona

van de Hulst H.C., 1957, Light Scattering by Small Particles. J. Wiley \& Sons, New York

Voshchinnikov N.V., 1990, SvA 34, 429

Voshchinnikov N.V., Farafonov V.G., 1993, Ap\&SS 204, 19

Voshchinnikov N.V., Il'in V.B., 1983a, Opt. Spectrosc. 55, 304

Voshchinnikov N.V., Il'in V.B., 1983b, SvA 27, 650

Voshchinnikov N.V., Il'in V.B., 1983c, SvA Lett. 9, 101

Waters L.B.F.M., Molster F.J., de Jong T., et al., 1996, A\&A 315, L361

Winters J.M., 1994, PhD Thesis, Techn. Univ., Berlin

Woitke P., Dominik C., Sedlmayr E., 1993, A\&A 274, 451 